\title{
Does the Milky Way have a Maximal Disk?
}

\author{
Penny D. Sackett \\ Institute for Advanced Study, Princeton, New Jersey 08540, USA \\ and \\ Kapteyn Astronomical Institute, 9700 AV Groningen, The Netherlands \\ psackett@astro.rug.nl
}

\begin{abstract}
The Milky Way is often considered to be the best example of a spiral for which the dark matter not only dominates the outer kinematics, but also plays a major dynamical role in the inner galaxy: the Galactic disk is therefore said to be "sub-maximal." This conclusion is important to the understanding of the evolution of galaxies and the viability of particular dark matter models. The Galactic evidence rests on a number of structural and kinematic measurements, many of which have recently been revised. The new constraints indicate not only that the Galaxy is a more typical member of its class (Sb-Sc spirals) than previously thought, but also require a re-examination of the question of whether or not the Milky Way disk is maximal. By applying to the Milky Way the same definition of "maximal disk" that is applied to external galaxies, it is shown that the new observational constraints are consistent with a Galactic maximal disk of reasonable $M / L$. In particular, the local disk column can be substantially less than the oft-quoted required $\Sigma_{\odot} \approx 100 \mathrm{M}_{\odot} \mathrm{pc}^{-2}$ — as low as $40 \mathrm{M}_{\odot} \mathrm{pc}^{-2}$ in the extreme case - and still be maximal, in the sense that the dark halo provides negligible rotation support in the inner Galaxy. This result has possible implications for any conclusion that rests on assumptions about the potentials of the Galactic disk or dark halo, and in particular for the interpretation of microlensing results along both LMC and bulge lines of sight.
\end{abstract}

Subject headings: Galaxy: structure — Galaxy: kinematics and dynamics — galaxies: kinematics and dynamics — galaxies: dark matter

Submitted to the Astrophysical Journal on 25 August 1996 Accepted for publication on 20 January 1997 


\section{Introduction}

The total amount and distribution of mass in disk galaxies determines their kinematics, and thus the amplitude and shape of their stellar and gaseous rotation curves. Since rotation curves of spirals fall much less rapidly than would be expected from the distribution of their light, it is generally inferred (assuming Newtonian gravity) that unseen, extended mass is present that dominates the kinematics in the outer regions of the disk. For both external galaxies and the Milky Way, however, the total amount of this "dark" mass within a given radius is ill-constrained, partially due to uncertainty in the geometry of the dark mass, and partially due to uncertainty in the fraction of the total mass supplied by the stellar disk itself (cf. Sackett 1996). Loosely speaking, if the disk is thought to dominate the mass in the inner regions of the galaxy, it is said to be "maximal," whereas if the dark halo is dynamically important in the inner galaxy the disk is said to be "sub-maximal."

It is important to settle whether spiral disks are maximal because the disk mass is coupled directly (through the rotation curve) to the radial distribution of halo dark mass, the form of which is predicted by models for galaxy formation and the nature of dark matter. In general, models of dissipationless dark matter predict that spiral disks should not be maximal: the dark mass in these models is substantially less flattened vertically than the disk baryons and has a small core radii so that it dominates even the inner galactic kinematics. For external spirals, evidence has been presented both for and against maximal disks (Casertano \& van Albada 1990; Freeman 1993; van der Kruit 1995), with most of the controversy centered on the uncertainty in the stellar disk mass (or more correctly, the total mass associated with the stellar disk). Since the local disk mass can be more directly measured in the Milky Way, the question has been less controversial for the Galaxy: it is generally believed that the Milky Way disk is substantially sub-maximal (Bahcall 1984; van der Kruit 1989; Kuijken \& Gilmore 1991, Merrifield 1993, Kuijken 1995).

Here we re-examine the evidence that has led to the suggestion that the Milky Way disk is not maximal, in light of new observations that could alter considerably the estimated contribution of the disk mass to the inner rotation curve of the Galaxy. Since the discussion of this point has sometimes been hindered by imprecision in the definition of "maximal," we define and apply a consistent definition of the term to both the Galaxy and external spirals. We will show that new observations of the structural parameters of the Milky Way disk suggest that the Galaxy is consistent with the structure inferred for external disks, and in particular with a "maximal" disk. Our approach differs from Sellwood \& Sanders (1988) in that we do not attempt to fit the uncertain Galactic rotation curve with a single disk model, but rather explore a variety of models consistent with the newer constraints. A definition

of maximal disk, as it is applied in external galaxies, is formulated in $\S 2$. In $\S 3$, recent 
observational determinations of the kinematic and structural parameters of the Galaxy are reviewed. In light of these new Galactic constraints, the maximum disk hypothesis is tested in the Milky Way in $\S 4$ and shown to be viable. Concluding remarks can be found in $\S 5$.

\section{What is a Maximal Disk?}

Stellar disks, including that of the Milky Way, are generally modeled as double exponential disks, with volume densities given by

$$
\rho(R, z)=\rho_{o} e^{-R / h_{R}} e^{-|z| / h_{z}}
$$

where $R$ and $z$ are the natural cylindrical coordinates of the axisymmetric disk, and $h_{R}$ and $h_{z}$ are the scale length and scale height of the disk, respectively. The integral column of disk mass (integrated to infinity) for such a disk at any galactocentric radius $R$ is $\Sigma(r)=\Sigma_{o} e^{-R / h_{R}}$, where $\Sigma_{o}=2 \rho_{o} h_{z}$ is the central (face-on) surface mass density. The total disk mass is then simply $M=2 \pi \Sigma_{o} h_{R}^{2}$.

The equatorial rotation curve due to this massive disk can be found by solving Poisson's equation for the corresponding gravitational potential $\Phi$, and then differentiating $\Phi$ to find the radial force giving rise to the circular speed at $R$. In this way, a general 2-D integral for the radial force of thick disks was computed by Casertano (1983); Kuijken \& Gilmore (1989a) reduced this to a 1-D integral for double exponential disks. With the assumption of circular orbits, the radial force leads directly to the rotation curve via Newton's second law. Although the rotation curve of a substantially thickened exponential disk peaks at slightly larger radius than that of an infinitely thin one, a thick disk curve is also less sharply peaked. The net result is that the speed of the disk at $R=2.2 h_{R}$ is equal to the peak rotation speed of the disk to $<1 \%$ over a wide range of scale heights, $h_{z}$. We will thus refer to $v^{\text {disk }}\left(2.2 h_{R}\right)$ as the maximum rotation speed of the disk.

The total circular speed of material at any point in a spiral galaxy is a result of the combined rotation support at that radius of the massive disk, halo, and bulge components. (Here "halo" is used to indicate dark halo; the observed metal-weak stellar halo contains so little mass as to be dynamically negligible.) Thus, in order to determine the radial distribution of halo dark mass from the rotation curve of a galaxy, the disk and bulge contributions must be well-understood and properly subtracted. In external galaxies, the structural parameters of the bulge and disk light can be measured directly via surface photometry. The conversion of these measurements into structural parameters for bulge and disk mass, however, is not uniquely determined. It is generally assumed that the mass-to-light ratios $(M / L)$ of the bulge and disk components do not vary with radius, in 
which case the luminous structural parameters of the bulge and disk control the shape of the bulge and disk rotation curves, with the single number, $M / L$, controlling the amplitude.

The "maximal disk hypothesis" is commonly stated as the hypothesis that the luminous disk alone is responsible for the circular velocity in the inner region of the rotation curve (van Albada \& Sancisi 1986). Strictly speaking, however, this definition is never applied to external galaxies for three reasons: (1) The luminous disk mass cannot be separated from dark disk mass with the same distribution (i.e., with the same $h_{R}$ ). (2) In galaxies with prominent bulges, the luminous bulge mass also contributes to the inner rotation support. (3) Since non-hollow halos are generally used for rotation curve fits, a small amount of inner rotation support must arise from the core of the halo itself.

In practice, then, the maximum disk hypothesis is imposed by assuming a functional form for the dark halo (isothermal with core is a common choice), and then fitting the entire rotation curve while adjusting the free halo parameters so as to accomodate the largest possible disk $M / L$. (If the galaxy has a prominent bulge, the bulge $M / L$ may be maximized first, or for simplicity set equal to the disk $M / L$.) This leads to a "maximum" disk mass that provides somewhat less than the total rotation support at $2.2 h_{R}$ where the disk rotation curve peaks, and substantially less support at larger radii where the dark halo is presumed to dominate.

For external galaxies with type similar to that of the Milky Way (Sb to Sc), such maximum disk fits produce massive disks whose peak disk rotation speed $v_{\max }^{\text {disk }}=v^{\text {disk }}\left(2.2 h_{R}\right)$, is $75 \%$ to $95 \%$ of the total circular speed at that radius (cf. Begeman 1987; Broeils 1992). The lower end of this range is occupied by galaxies with large bulges or bars that contribute significantly to $v\left(2.2 h_{R}\right)$, thus decreasing the importance of the disk in the inner regions. In general, the total circular speed at $R=2.2 h_{R}$ may be somewhat different than the peak rotation speed of the galaxy as a whole, depending on whether the rotation curve falls slightly (typical for massive bulge-dominated spirals) or rises (typical for extreme late-type spirals), but for Sb-Sc spirals the two are comparable. In very late-type or dwarf spirals, a significant fraction of the rotation support may be provided by the mass of the neutral gas, but this is negligible in the inner portion of Sb-Sc spirals.

In summary, the definition of maximum disk that is typically used in external spirals does not necessarily require that the detected luminous disk matter provide all the rotation support in the inner galaxy. If spirals contain substantial disk dark mass with a scale length similar to that of the light, the disk rotation curve shape will remain unchanged, but the inferred $M / L$ ratio of the massive disk will be larger than the integrated stellar $M / L$ of the disk. Secondly, a maximum disk fitted to the rotation curve of an external spiral with a bulge component may supply as little as $75 \%$ of $v\left(2.2 h_{R}\right)$ and thus contain only slightly 
more than one-half of the mass interior to that radius. The practical application of the maximum disk hypothesis to spirals similar to the Milky Way produces disks that provide $85 \% \pm 10 \%$ of the total rotation support of the galaxy at $R=2.2 h_{R}$. Rather than attempt a multi-component fit to the uncertain Galactic rotation curve, this extragalactic working definition of maximal disk will be applied directly to current observations of the Milky Way.

\section{Observational Constraints on the Structure of the Galactic Disk}

In external galaxies, the rotation curve and scale length of the disk light are generally well-determined, but the mass normalization of the disk is poorly constrained. In the Milky Way, the situation is reversed. The local surface mass density of the Galaxy is well-constrained by kinematical studies of old stars in the solar neighborhood, although parceling this mass column into halo and disk components has proven less straightforward. As in external galaxies, it is generally assumed that the luminous and massive disk have the same scale length, but in the Milky Way determinations of the luminous scale length are hindered by our observing vantage point, which places us in the middle of the dusty disk. The rotation support supplied by the massive disk must be compared to the observed rotation curve for the Galaxy, but here too our position inside the disk introduces uncertainties and ambiguities that plague attempts to construct an accurate rotation curve. Finally, in order to convert measurements of the circular rotation referenced to the Local Standard of Rest (LSR) and local surface mass density into an absolute rotation curve and total disk mass, an accurate measurement of our distance from the Galactic Center is required.

In the following sections, new determinations of important structural and kinematic properties of Milky Way will be discussed in turn: local surface mass density, $\Sigma_{\odot}$; scale parameters of the luminous disk, $h_{R}$ and $h_{z}$; the distance to the center of the Galaxy, $R_{o}$; the rotation curve, $v(R)$; and local circular speed, $v_{o}$. As we shall see, these crucial measurements have evolved over the past few years in a direction that requires a reassessment of whether the Galactic disk is maximal. A short note on the bulge of the Milky Way and its effect on this analysis ends this section.

\subsection{Local Surface Mass Density}

The distances and kinematics of old, resolved stars can be used to measure the vertical restoring force of the local Galactic disk — and thus its surface mass density. By measuring 
the velocities of local stars as a function of height above the Galactic plane, Kuijken \& Gilmore (1991, KG) report a total mass column of $\Sigma_{1.1 \mathrm{kpc}}=71 \pm 6 \mathrm{M}_{\odot} \mathrm{pc}^{-2}$, integrated within a $1.1 \mathrm{kpc}$ band above and below the plane. This is a rather robust measurement that depends only weakly on assumed scale parameters for the disk. The separation of this surface mass density into disk and halo components, however, is much more dependent on the assumed scale length $h_{R}$ and scale height $h_{z}$ of the disk, and on the distance $R_{o}$ to the Galactic center. Assuming $h_{R}=4.5 \mathrm{kpc}, h_{z}=0.3 \mathrm{kpc}$, and $R_{o}=7.8 \mathrm{kpc}, \mathrm{KG}$ concluded that $\Sigma_{\odot}=48 \pm 9 \mathrm{M}_{\odot} \mathrm{pc}^{-2}$ was due to the disk itself, with the rest contributed by a rounder dark halo. Gould's reanalysis (1990) of the original Kuijken \& Gilmore dataset (1989b) weighs in at a similar $\Sigma_{\odot}=54 \pm 8 \mathrm{M}_{\odot} \mathrm{pc}^{-2}$.

If the local dark matter contains a significant disk-like component, these numbers would increase. Using a different dataset than that of $\mathrm{KG}$, and fitting models in which the local dark matter distribution is proportional to the disk luminous matter (assuming $h_{R}$ $=3.5 \mathrm{kpc}, h_{z}=0.35 \mathrm{kpc}$, and $R_{o}=8 \mathrm{kpc}$ ) Bahcall (1984) suggested that the total local disk column may be as much as $67 \mathrm{M}_{\odot} \mathrm{pc}^{-2}$. Applying the same models to newer data, Bahcall, Flynn \& Gould (1992) derived an even larger total disk column at the Sun of $84_{-24}^{+29} \mathrm{M}_{\odot} \mathrm{pc}^{-2}$, although a recent redetermination of the normalization of the local density of the kinematical tracer population later led Flynn \& Fuchs (1994) to revise this estimate downwards to $56 \pm 10 \mathrm{M}_{\odot} \mathrm{pc}^{-2}$. If the dark matter is disk-like but with a scale height larger than that of the luminous matter, the total disk column may be somewhat higher than these estimates. A strict lower limit on the local surface mass density is given by the total column of directly observed material in the solar neighborhood. The lowest recent estimate is that of Gould, Bahcall \& Flynn (1996) who report that the column of M dwarfs inferred from their deep HST observations is smaller than previously thought, yielding a total local observed column of $\Sigma_{\odot, \text { obs }}=39 \mathrm{M}_{\odot} \mathrm{pc}^{-2}$.

Independent recent measurements thus seem to confirm that the local surface mass density of the thin disk is about $\Sigma_{\odot}=53 \pm 13 \mathrm{M}_{\odot} \mathrm{pc}^{-2}$, with a weak dependence on the assumed structural parameters of the luminous disk and dark matter distribution. As stressed by $\mathrm{KG}$, the more robust estimate is $\Sigma_{1.1 \mathrm{kpc}}=71 \pm 6 \mathrm{M}_{\odot} \mathrm{pc}^{-2}$ for the total local column between $1.1 \mathrm{kpc}$ above and below the plane.

\subsection{Scale Lengths and Heights of the Thin and Thick Disks}

Disk scale lengths $h_{R}$ of 3.5 and $4.5 \mathrm{kpc}$ have been used recently by authors studying the disk mass distribution (cf. Bahcall, Flynn \& Gould 1992; Kuijken \& Gilmore 1989b, 1991). The newer observations which we now discuss, however, suggest that the scale length 
of the disk stars in the Milky Way may be considerably shorter. Since measurements of $h_{R}$ scale with the solar distance $R_{o}$, the assumed $R_{o}$ is listed in Table 1 for each determination of $R_{o}$. As we will see in $\S 4$, the dynamical importance of the disk is sensitive to the ratio $R_{o} / h_{R}$, which is more observationally secure than $h_{R}$.

The three-dimensional distribution of 2.4 micron light from SpaceLab was used by Kent, Dame \& Fazio (1991) to derive a scale length for the Galactic disk of $h_{R}=3.0 \pm 0.5 \mathrm{kpc}$. They derive a scale height of IR light $h_{z}=0.247 \mathrm{kpc}$ at the solar position, decreasing to $h_{z}=0.165 \mathrm{kpc}$ at a distance of $5 \mathrm{kpc}$ from the Galactic center. Kent, Dame \& Fazio review earlier work on the disk scale length $h_{R}$ and note that values as small as $1.8 \mathrm{kpc}$ and as large as $6.0 \mathrm{kpc}$ have been suggested. Infrared estimates based on integrated light or star counts tend to give shorter scale lengths than their optical counterparts. This mirrors the situation in external galaxies in which longer scale lengths are found in bluer bands, perhaps indicative of radial gradients in age and metallicity (cf. de Jong 1996). Measurements in redder bands have the advantage that they are more likely to reflect the distribution of stellar mass (since they are dominated by the emission from low-mass stars that in turn dominate the stellar mass) and are less contaminated by extinction from dust, especially important in edge-on systems (like the Milky Way).

A review of early determinations of Galactic structure constants based on optical star count studies prior to 1990 can be found in Robin, Crézé, \& Mohan (1992). Although these earlier optical studies supported moderate scale lengths of $3.5-4.5 \mathrm{kpc}$ (as does the single-field study of $\mathrm{Ng}$ et al. 1995), Robin, Crézé, \& Mohan found evidence for much shorter scale length of $h_{R}^{\text {thin }}=2.5 \pm 0.3 \mathrm{kpc}$ and a sharper decrease in counts beyond $6 \mathrm{kpc}$. Most of the more modern determinations of $h_{R}$ from optical star count studies are smaller than the pre-1990 optical estimates and closer to IR-estimated scale lengths. By modeling local kinematic data using an approximation that the stellar disk is cold and self-gravitating, Fux \& Martinet (1994) concluded that the old disk has a scale length of $2.5_{-0.6}^{+0.8} \mathrm{kpc}$, under the assumption of a constant scale height. If they instead assumed an inwardly decreasing scale height, the best-fitting scale length increased to $3.1 \mathrm{kpc}$. Using UBVR photometry and proper motions from three fields in the direction of the Galactic center, anti-center, and anti-rotation, combined with previous wide-field surveys, Ojha et al. (1996) derived the Galactic stellar density as a function of position and magnitude interval. From this, they derive scale lengths and heights of $h_{R}^{\text {thin }}=2.3 \pm 0.6 \mathrm{kpc} ; h_{z}^{\text {thin }}=0.26 \pm 0.05 \mathrm{kpc}$ and $h_{R}^{\text {thick }}=3 \pm 1 \mathrm{kpc} ; h_{z}^{\text {thick }}=0.76 \pm 0.05 \mathrm{kpc}$ for the thin and thick disk components of the Galaxy, respectively. These results are in excellent agreement with those of Robin, Crézé, \& Mohan (1992) for the thin disk and Robin et al. (1996) who, on the basis of a maximum likelihood analysis of a variety of Galactic field samples from a variety of authors find $h_{R}^{\text {thick }}=2.8 \pm 0.8 \mathrm{kpc}$, and $h_{z}^{\text {thick }}=0.76 \pm 0.05 \mathrm{kpc}$. In a very recent study using a 
deep HST sample, Gould, Bahcall \& Flynn (1996) report an M-dwarf disk scale length of $h_{R}=3.0 \pm 0.4 \mathrm{kpc}$. Their sample is most sensitive to stars far from the plane $(\sim 2 \mathrm{kpc})$. The work of Reid \& Majewski (1993) suggests that the thin and thick disk scale heights may be considerably larger than previously thought: they obtain $h_{z}^{\text {thin }}=0.4 \mathrm{kpc}$ for less luminous (M-dwarf) tracers, and $h_{z}^{\text {thick }}=1.5 \pm 0.1 \mathrm{kpc}$. These authors also review the previous literature by others on the thick disk scale height, which typically lie in the lower range $0.7<h_{z}^{\text {thick }}<1.2 \mathrm{kpc}$.

In summary, recent evidence from a variety of studies drawing on different datasets suggests that the scale length of the old, thin disk is in the range $h_{R}^{\text {thin }}=2.5 \pm 0.5 \mathrm{kpc}$. If the scale height of the disk decreases inwards, but is modeled as remaining constant, estimates for $h_{R}$ may be biased artificially downwards, in which case the thin disk scale length may be more nearly $h_{R}^{\text {thin }}=3.0 \pm 1 \mathrm{kpc}$. The thick disk scale length $h_{R}^{\text {thick }}$ is probably similar to $h_{R}^{\text {thin }}$, though less well-constrained. Recent determinations of $h_{R}$ for the Galaxy are consistent with observations of external galaxies of the same type: the median scale length for Sb-Sc spirals derived from de Jong's (1996) total sample of 86 face-on galaxies lies between 2.7 and $3.3 \mathrm{kpc}$ (for $H_{0}=75 \mathrm{~km} \mathrm{~s}^{-1} \mathrm{Mpc}^{-1}$ ), while the median scale length for Courteau's sample (1996) of over 300 spirals of varying inclination is $3.9 \mathrm{kpc}$ for the same $H_{0}$ (1997, private communication). Galactic measurements of $h_{R}$ are relative measurements that generally scale with the assumed distance solar distance $R_{o}$. As we will see, the dynamical importance of the disk is most sensitive to the ratio $R_{o} / h_{R}$. Finally, most estimates for the local scale heights of the thin and thick components indicate that $h_{z}^{\text {thin }}=0.30 \pm 0.05 \mathrm{kpc}$ and $h_{z}^{\text {thick }}=1 \pm 0.25 \mathrm{kpc}$, respectively.

\subsection{Distance to the Galactic Center}

An excellent review of recent determinations of the distance $R_{o}$ to the Galactic Center has been compiled by Reid (1993) and need not be repeated here. Reid concludes that the weight of the evidence suggested that $R_{o}=8.0 \pm 0.5 \mathrm{kpc}$, with the error representing

both systematic and statistical uncertainties. We will assume this range for $R_{o}$ here, which includes the current IAU standard value of $8.5 \mathrm{kpc}$ as its upper limit.

\subsection{The Rotation Curve of the Galaxy}

Whereas the rotation curves of external galaxies are relatively straight forward to measure, the rotation curve of the Galaxy has proven notoriously difficult to constrain (see 
reviews by Fich \& Tremaine 1991, Merrifield 1993; Schechter 1993). A review of all recent determinations of $v_{o}$ is beyond the scope of this article, we concentrate on those works that illustrate the nature of the discrepancy remaining in this important Galactic measurement.

Interior to the solar position at $R_{o}$, the tangent point (or terminal velocities) method produces accurate measurements (if the orbits are circular), but the velocities are measured relative to the local velocity $v_{o}$ and distances are expressed in units of $R_{o}$. In order to convert these data into an absolute rotation curve, assumptions must be made for the solar distance and local circular speed; the IAU standards of $R_{o}=8.5 \pm 1.1 \mathrm{kpc}$ and $v_{o}=220 \pm 20 \mathrm{~km} \mathrm{~s}^{-1}$, based on a review by Kerr \& Lynden-Bell (1986), have been typical choices in the past. Most recent estimates for both the local circular speed and distance to the Galactic center tend to be lower than the 1986 IAU standards. In the inner galaxy, estimates from the tangent point method yield a considerably lower value for $2 A R_{o} \equiv v_{o}-R_{o}\left(d v_{c} / d R\right)_{R_{o}} \equiv-R_{o}{ }^{2}(d \Omega / d R)_{R_{o}}$, where $A$ is Oort's constant and $\Omega$ is the disk angular velocity. Comparing to previous analyses, this suggests one or more of the following: $v_{o}$ is smaller; $R_{o}$ is smaller; or the local rotation curve turns over less fast (i.e., the Oort constant $\mathrm{A}$ is lower).

Using the tangent point method on the neutral gas (H I) component together with their new estimates for the solar distance and Oort constants, Rohlfs et al. (1986) derived a rotation curve that dropped from $200 \mathrm{~km} \mathrm{~s}^{-1}$ at $6 \mathrm{kpc}$ to $170 \mathrm{~km} \mathrm{~s}^{-1}$ at $10 \mathrm{kpc}$, and flattened to $200 \mathrm{~km} \mathrm{~s}^{-1}$ again at large radii. The outer rotation curve was determined from $\mathrm{H}$ II regions. Their best fit value of $v_{o}=184 \mathrm{~km} \mathrm{~s}^{-1}$. They noted that their lower estimate for $v_{o}$ actually produces a rotation curve for the Milky Way whose amplitude and slope more nearly matches that of external Sb-Sc spirals. Larger values produced an atypical steeply-rising curve.

Merrifield (1992) used an axisymmetric model of the neutral hydrogen layer to fit the Galactic H I kinematics as a function of longitude and latitude, solving simultaneously for the form of the rotation curve (scaled to the solar distance and LSR speed) and the scale height of the gas as a function of radius. He was able to use this method out to $2.5 R_{o}$, with different choices for $v_{o}$ and $R_{o}$ leading to different slopes for the outer rotation curve. Using the Oort constants of Kerr \& Lynden-Bell (1986), he finds that his data requires $R_{o}=7.9 \pm 0.8 \mathrm{kpc}$ and $v_{o}=210 \pm 25 \mathrm{~km} \mathrm{~s}^{-1}$. In order to be consistent with the rotation curve slopes of external galaxies of the same type, Merrifield $(1992,1993)$ concluded that $v_{o}=180 \pm 20 \mathrm{~km} \mathrm{~s}^{-1}$. (Larger $v_{o}$ or smaller $R_{o}$ will cause a sharp increase in the slope of the rotation curve, as noted by Rohlfs et al. 1986.) Merrifield thus compromised on $v_{o}=200 \pm 10 \mathrm{~km} \mathrm{~s}^{-1}$.

In the outer Galaxy tangent points are not available, and the kinematics of standard 
candles are typically used, which are generally less reliable. These measurements tend to give higher values for $v_{o}$; though this may in part be due to (slight) disk ellipticity.

A recent determination from Cepheid work (Pont, Major \& Bruki 1994) measures the solar distance and Oort constants, and variation of the Cepheid kinematics with distance. Together these allow a determination of Galactic rotation curve (which must be normalized with $v_{o}$ ). Their solar distance of $R_{o}=8.09 \pm 0.3 \mathrm{kpc}$ falls nicely within other determinations, but their rather high Oort constant yields $2 A R_{o}=257 \pm 7 \mathrm{~km} \mathrm{~s}^{-1}$, which for a flat rotation curve would result in a very large $v_{o}=257 \mathrm{~km} \mathrm{~s}^{-1}$. Since their Cepheid rotation curve appears to fall in the region of the solar radius, however, the implied local circular speed $v_{o}$ would probably be lower, by an amount that depends the gradient $\left(d v_{c} / d R\right)_{R_{o}}$. Using their Oort constant $A$, but current IAU standards for $R_{o}$ and $v_{o}$, Pont et al. (1996) find that their outer rotation curve is flat at $200 \mathrm{~km} \mathrm{~s}^{-1}$. They note that it is possible that their rotation curve is miscalibrated due to a systematic effect caused by metallicity gradients in the Cepheids with Galactocentric distance, and plan to design new observations to test this hypothesis. Such metallicity effects can lead to incorrect extinction corrections and thus incorrect Cepheid distances (Stothers 1988, Gould 1994, Beaulieu et al. 1996).

Schechter (1993) reports that carbon star kinematics in the outer Galaxy are consistent with a flat rotation curve of $227 \mathrm{~km} \mathrm{~s}^{-1}$, with at least $10 \%$ uncertainty from the uncertainty in the Local Standard of Rest (LSR) angular speed about the Galactic Center. This uncertainty may decrease soon as the VLA proper motions of Sgr A*, which is believed to be stationary at the Galactic Center, improve with time. Backer (1996) reports an observed proper motion of $\operatorname{Sgr} \mathrm{A}^{*}$ in the longitudinal and latitudinal directions of $-6.55 \pm 0.34$ mas yr$^{-1}$ and $-0.48 \pm 0.23$ mas yr$^{-1}$, respectively. (Note that these are $2 \sigma$ errors.) This must be corrected to account for the motion of the Sun with respect to the LSR (Mihalas \& Binney 1981) tangent to the Galactic rotation of $+12 \mathrm{~km} \mathrm{~s}^{-1}$. For $R_{o}=8.0 \mathrm{kpc}$, this leads to a local circular speed of $236 \pm 13 \mathrm{~km} \mathrm{~s}^{-1}$, considerably higher than estimates from the kinematics of $\mathrm{H}$ I in the disk.

Kuijken \& Tremaine (1994) suggest that some of the discrepancy between $v_{o}$ as determined from stellar and $\mathrm{H}$ I tracers at different positions may be due to ellipticity of the galactic potential, and can be rectified if the disk isopotential curves are slightly elliptic to yield a true circular speed of about $v_{o}=200 \mathrm{~km} \mathrm{~s}^{-1}$. We conclude that the circular speed at the solar radius is probably lower than the standard IAU value and instead lies in the range $v_{o}=210 \pm 25 \mathrm{~km} \mathrm{~s}^{-1}$ which, assuming that $R_{o} \approx 8 \mathrm{kpc}$, encompasses the best values from the $\mathrm{H}$ I analysis of $185 \mathrm{~km} \mathrm{~s}^{-1}$ on the low side and estimates based on the Sgr $\mathrm{A}^{*}$ proper motion of $235 \mathrm{~km} \mathrm{~s}^{-1}$ on the high side. 


\subsection{A Word about the Bulge}

Sellwood \& Sanders (1988) were able to construct a maximum disk model for the Galaxy by assuming a disk scale length of $4 \mathrm{kpc}$ and a very massive bulge of $(3-4) \times 10^{10} \mathrm{M}_{\odot}$. More recent estimates suggest that the bulge has a somewhat lower mass at $(1-2) \times 10^{10} \mathrm{M}_{\odot}$, and is likely, in fact, to be bar-like (see Zhao, Spergel \& Rich 1995 for a review), making a maximum disk solution less viable. On the other hand, the smaller Milky Way scale length indicated by more recent measurements operates in the other direction, as we shall see in $\S 4$. Note that the effect of the bulge is already taken into account by our definition of maximal through the spread in the fractional rotation support $(85 \pm 10 \%)$ provided by external maximal disks at $2.2 h_{R}$ - the more massive the bulges, the smaller the required disk support. The primary results of the next section thus do not depend on a specific bulge model. Given the uncertainty in the morphology and mass of the Galactic bulge, we do not draw any conclusions about bulge parameters, but do give one example a simple, illustrative model of the Galactic bulge to indicate roughly the magnitude of its effect on Milky Way kinematics.

\section{A Maximum Disk in the Milky Way}

The Milky Way is usually said to have a sub-maximal disk (Bahcall 1984; Kuijken \& Gilmore 1989b; van der Kruit 1989, Kuijken \& Gilmore 1991, Kuijken 1995). This statement is based on the observation that an exponential disk with a local surface mass density of $\Sigma_{\odot} \approx 50 \mathrm{M}_{\odot} \mathrm{pc}^{-2}$ at an assumed $8<R_{o}<8.5 \mathrm{kpc}$, and a scale length $3.5<h_{R}<4.5 \mathrm{kpc}$ can provide only about half of the IAU-accepted local circular rotation speed of $v_{o}=220 \mathrm{~km} \mathrm{~s}^{-1}$. To provide all of the IAU estimate for $v_{o}$, an exponential disk of this $h_{R}$ would need to have a local mass column of $\Sigma_{\odot} \approx 100 \mathrm{M}_{\odot} \mathrm{pc}^{-2}$.

A more uniform definition of the "maximum disk hypothesis," however, together with revised estimates for Galactic structure constants requires a reanalysis of this conclusion:

- In external galaxies, the fraction of disk support is usually computed at the point where the disk rotation curve peaks at $\sim 2.2 h_{R}$ (which need not equal $R_{o}$ in the Galaxy), or through fits to the entire rotation curve.

- Massive bulges and non-hollow halos require that a disk with maximum mass actually provides less than $100 \%$ of $v\left(2.2 h_{R}\right)$ : maximal disk fits in external Sb-Sc spirals typically give $v^{\text {disk }}\left(2.2 h_{R}\right) / v\left(2.2 h_{R}\right)=85 \pm 10 \%$. The Milky Way is known to have a massive bulge or bar, though its precise dynamic contribution is ill-constrained. 
- The total local column of the thin disk mass is probably $\Sigma_{\odot}=53 \pm 13 \mathrm{M}_{\odot} \mathrm{pc}^{-2}$, but the column within $1.1 \mathrm{kpc}, \Sigma_{1.1 \mathrm{kpc}}=71 \pm 6 \mathrm{M}_{\odot} \mathrm{pc}^{-2}$, is more robust. A column of $50 \pm 10 \mathrm{M}_{\odot} \mathrm{pc}^{-2}$ is probably appropriate for the total observed then disk at $R_{o}$.

- Recent estimates for the scale lengths of the (old) thin and thick disks remain somewhat uncertain, but are lower than previous estimates; $h_{R}=3.0 \pm 1 \mathrm{kpc}$ is now an appropriate range. Estimates for the scale heights vary, but are generally bracketed by $h_{z}^{\text {thin }}=0.30 \pm 0.05 \mathrm{kpc}$ and $h_{z}^{\text {thick }}=1 \pm 0.25 \mathrm{kpc}$. A range of plausible disk $h_{z}$ must be considered because rotation curve fitting in external galaxies is unable to place constraints on the scale height of the disk mass.

- New determinations for solar distance $R_{o}$ are somewhat lower than the 1986 IAU standard of $8.5 \mathrm{kpc}$, with $8.0 \pm 0.5 \mathrm{kpc}$ providing a plausible range.

- The latest determinations from both the H I velocity field and varying H I scale height of the neutral gas in the Milky Way indicate that the Galactic rotation curve has a local circular speed that is considerably lower than the IAU value of $220 \mathrm{~km} \mathrm{~s}^{-1}$, but stellar tracers still tend to give higher values. We choose $v_{o}=210 \pm 25 \mathrm{~km} \mathrm{~s}^{-1}$ as a plausible range. Note that use of the Tully-Fisher relation (Pierce \& Tully 1988; Jacoby et al. 1992) indicates that this velocity range corresponds to a total luminosity for Milky Way of $L_{V} \approx\left(2.1_{-0.6}^{+1.0}\right) \times 10^{10} L_{\odot, V}$, consistent with observational estimates of $\left(1.4<L_{V}<2.0\right) \times 10^{10} L_{\odot, V}$ (van der Kruit 1986; Binney \& Tremaine 1987), with velocities in the lower end of the range matching somewhat better.

Our goal is not to test any one choice for Galactic structure parameters, nor to argue that one set is preferable to another, but rather to ask whether the maximum disk hypothesis - as it is applied in external galaxies - is consistent with the newest constraints on the range of likely structural parameters for the Galaxy. We will do this by computing the rotation support at $2.2 h_{R}$ supplied by various disk models as a function of the assumed ratio of solar distance to disk scale length $R_{o} / h_{R}$, and then asking whether this rotation support lies within $85 \pm 10 \%$ of the rotation speed at that distance. The parameter $R_{o} / h_{R}$ is important because the maximum speed supplied by the disk is proportional to $\sqrt{M_{\text {disk }}}$, and the disk mass is normalized through a local measurement of the total column of disk mass through $M_{\text {disk }}=2 \pi \Sigma_{\odot} h_{R}^{2} e^{R_{o} / h_{R}}$. We assume here that the rotation curve is approximately flat between $R=2.2 h_{R}$ and $R=R_{o}$ so that $v\left(2.2 h_{R}\right) \approx v\left(R_{o}\right) \equiv v_{o}$, and that any slight slope between these radii lies within the uncertainty in $v_{o}$. 


\subsection{The Old Definition and Old Constraints for a Galactic Maximal Disk}

Shown in Fig. 1 is the rotation support supplied by the disk at the solar position ( $R=R_{o}$ ) as a function of $R_{o} / h_{R}$ for a variety of disk mass models and assumptions for the solar distance $R_{o}$. Three mass models, none of which may be correct in detail, but which reasonably span the model space are considered: (a) Very thin, very light disk: the total mass column of the disk at $R_{o}$ is set equal to the minimum estimate of $\Sigma_{\odot}=40 \mathrm{M}_{\odot} \mathrm{pc}^{-2}$ corresponding to a lower limit on the directly observed components alone, with a small scale height of $h_{z}=0.25 \mathrm{kpc}$ (solid lines). (b) Moderately thin, heavy disk: the disk mass is assumed to follow the distribution of thin disk light with a scale height $h_{z}=0.35 \mathrm{kpc}$, using an upper limit for the measured local column of thin disk mass between $1.1 \mathrm{kpc}$ of $\Sigma_{1.1 \mathrm{kpc}}=65 \mathrm{M}_{\odot} \mathrm{pc}^{-2}$ (dashed lines). (c) Thick, heavy disk: the disk mass is assumed to follow the distribution of thick disk light with a scale height $h_{z}=1.0 \mathrm{kpc}$, and a local column of disk mass between $1.1 \mathrm{kpc}$ equal to $\Sigma_{1.1 \mathrm{kpc}}=75 \mathrm{M}_{\odot} \mathrm{pc}^{-2}$ (dotted lines). The rotation curve for each model was calculated in the manner described in $\S 2$, taking into full account the thickness of the disk.

For each mass model, two extreme choices for $R_{o}$ are indicated: a small distance to the Galactic center of $R_{o}=7.5 \mathrm{kpc}$, and the IAU standard value of $R_{o}=8.5 \mathrm{kpc}$, which now appears to lie at the upper end of the probable range. At fixed $R_{o} / h_{R}$, models with larger $R_{o}$ must also have larger $h_{R}$, and thus larger total disk mass and larger rotation support $\left(v \propto \sqrt{R_{o}}\right.$ for fixed $R_{o} / h_{R}$ and $\left.\Sigma_{\odot}\right)$. Models with higher local surface mass density have, naturally, a higher total mass at fixed $R_{o} / h_{R}$, and thus more rotation support $\left(v \propto \sqrt{\Sigma_{\odot}}\right.$ for fixed $R_{o} / h_{R}$ and $R_{o}$ ). Moving along a given model line toward increasing $R_{o} / h_{R}$ is equivalent to decreasing the disk scale length $h_{R}$ for that model. The disk support increases for smaller $h_{R}$ at fixed $R_{o}$ because the constant local normalization for the disk column then occurs at a larger number of disk scale lengths; this has an exponential effect on total disk mass that more than offsets the decrease in $M_{\text {disk }}$ with decreasing $h_{R}\left(v \propto \sqrt{h_{R} e^{1 / h_{R}}}\right.$ for fixed $R_{o}$ and $\left.\Sigma_{\odot}\right)$. Disk models that are constrained by a local column within $1.1 \mathrm{kpc}$ are more massive than those constrained to have the same total column, and thus produce larger disk support at the same $R_{o} / h_{R}$.

Fig. 1 shows how the maximum disk hypothesis has been tested previously in the Milky Way: the rotation support of a particular Galactic disk model at $R=R_{o}$ is compared to the IAU standard value of $v_{o}=220 \mathrm{~km} \mathrm{~s}^{-1}$. The popular models of Kuijken \& Gilmore (1991, KG) and Bahcall \& Soneira (1980, BS, with $\Sigma_{\odot}$ normalization from Bahcall 1984) are indicated in Fig. 1; both clearly fall far short of providing all of IAU standard circular speed. Indeed, this is the primary basis for previous statements that the Galactic disk is approximately "half-maximal." 


\subsection{Application of the New Definition}

Fig. 2 indicates the situation changes when using the extragalactic definition of maximal disk, which makes the comparison at $R=2.2 h_{R}$, (not $R=R_{o}$ ), and more recent Galactic constraints. The disk rotation support is now computed at its maximum at (i.e., $R=2.2 h_{R}$ ), and plotted in Fig. 2 for comparison with the speed that the disk must have in order to be considered maximal by the definition used in extragalactic studies (see $\S 2$ ). The ordinate bounds of the box in Fig. 2 indicate the upper and lower limits of the current observational constraints on the local circular speed $v_{o}=210 \pm 25 \mathrm{~km} \mathrm{~s}^{-1}$ multiplied by the fraction $85 \pm 10 \%$ of typical maximum disk support in external Sb-Sc galaxies at $2.2 h_{R}$. The abscissa bounds of the box indicate the constraints on $R_{o} / h_{R}$ (see Table 1 ). Considering the observational errors, $2<R_{o} / h_{R}<4.8$, but note that the best fit value of all recent determinations cluster in the range $2.7<R_{o} / h_{R}<3.5$. Models falling inside the box are simultaneously "maximal" and satisfy observational constraints on $R_{o} / h_{R}$; those above the box are too massive and those below are sub-maximal. The dynamical constraints actually rule out certain combinations of $R / h_{R}$ for the Milky Way that are allowed by the structural constraints alone. In particular, $R_{o} / h_{R}>4.3$ is excluded since then all disk models would be so massive as to exceed estimates for the local rotation speed, even without the addition of mass components such as the bulge and dark halo.

Models constrained to the "maximal disk box" have total disk masses that vary with $R_{o} / h_{R}$ and lie in the range: $3.1 \times 10^{10}<M<6.7 \times 10^{10} \mathrm{M}_{\odot}$ for the very thin, very light disk, $4.4 \times 10^{10}<M<8.0 \times 10^{10} \mathrm{M}_{\odot}$ for the moderately thin, heavy disk, and $7.3 \times 10^{10}<M<1.1 \times 10^{11} \mathrm{M}_{\odot}$ for the thick, heavy disk. Using observational determinations of the disk $\mathrm{V}$ band luminosity of the Galactic disk of $\sim 1.5 \times 10^{10} L_{\odot, V}$ (van der Kruit 1986; Binney \& Tremaine 1987), the full range of the implied mass-to-light ratios for these maximal disk models is thus $2 \lesssim M / L_{V} \lesssim 7$, which compares quite well with typical values of $2 \lesssim M / L_{V} \lesssim 6$ found for maximum disks of external Sb-Sc spirals (Begeman 1987; Broeils 1992; Broeils \& Courteau 1997).

All the disk models presented in Fig. 2 could be maximal if $R_{o} / h_{R} \geq 3$ (i.e., the scale length of the disk mass is $h_{R} \lesssim 2.5 \mathrm{kpc}$ ), which is within the observational constraints. The heavier disk models, those with larger disk scale height, and those assuming a larger solar distance, can be maximal for larger values of $h_{R}$. The KG model still falls below the Galactic maximum disk box; but also just to the left, indicating that their assumed $R_{o} / h_{R}$ may no longer be realistic. If the KG model were changed only by reducing the assumed disk scale length from $4.5 \mathrm{kpc}$ to $3 \mathrm{kpc}$, it could be a maximum disk model. The BS model clearly lies within the maximum disk box; changing their assumed $h_{R}$ from $3.5 \mathrm{kpc}$ to $3 \mathrm{kpc}$ would place close to the middle of the velocity limits. 


\subsection{Addition of an Illustrative Bulge Model}

Rather than model the bulge directly, the analysis of the previous section used as a constraint the range of rotation support supplied by the maximum disks of external Sb-Sc spirals, namely $85 \pm 10 \%$ of the total $v\left(2.2 h_{R}\right)$. This range encompasses the range of bulge masses typically seen in spirals similar in type to the Galaxy, and thus represents a reasonable range for the Milky Way. If the mass and profile of the Galactic bulge were known precisely, tighter constraints could be placed, but the structure of the bulge is a matter of some debate (e.g., Zhao, Spergel \& Rich 1995), and so we have chosen not to model it directly. Nevertheless, in order to assess (roughly) the dynamical influence of the bulge on the possibility of a Galactic maximal disk we now turn to a simple, empirical model for the bulge.

A detailed mass model of the Galaxy would likely include separate components for the Galactic Center, inner bulge, bar, thick disk, and metal-weak halo. Since the combined uncertainty in the structure of these components is large and controversial, we prefer to use the spherical spheroid model of de Vaucouleurs \& Pence (1978), which has a simple analytic form and is meant to account for all the non-disk (thin disk) light in the Galaxy. For illustrative purposes, we therefore consider a spheroidal mass component with an $R^{1 / 4}$ profile, and an effective radius and total mass scaled to give $R_{e}=2.67 \mathrm{kpc}$ and $M_{\text {bulge }}=1.5 \times 10^{10} \mathrm{M}_{\odot}$, respectively, for an assumed solar distance of $R_{o}=8 \mathrm{kpc}$. Models with different choices for $R_{o}$ are scaled appropriately.

In Fig. 3, the rotation support of this scaled bulge model has been added to that of each of the disk models to arrive at an estimate of total rotation supplied by the mass associated with the luminous components of the Milky Way at $R=2.2 h_{R}$. The ordinate bounds of the maximal disk box have now been changed to reflect $100 \%$ of the observational limits on the Galactic rotation curve. Again we see that all disk models can be considered maximal for some (allowable) range of Galactic parameters, and the models nicely bracket the most probable range of $R_{o} / h_{R}$ indicated by the smaller inset box. The heavy, thin disk model, for example, with $h_{z}=0.35 \mathrm{kpc}$ and a local mass column between $1.1 \mathrm{kpc}$ of $\Sigma_{1.1 \mathrm{kpc}}=65 \mathrm{M}_{\odot} \mathrm{pc}^{-2}$ (or total column $\Sigma_{\odot}=68 \mathrm{M}_{\odot} \mathrm{pc}^{-2}$ ), could be maximal over the full range of the median $R_{o} / h_{R}$ from recent Galactic observations, in the sense that the dark halo supplies none of the rotation support at $R=2.2 h_{R}$. 


\section{Conclusions and Implications}

Recent determinations for both the Galactic disk scale length, and the magnitude and slope of the rotation curve at the solar position are smaller than older values and more comparable to those determined for external Sb-Sc spirals, indicating that the Milky Way disk may provide more dynamical support in the inner Galaxy than previously thought. The maximum disk hypothesis applied to external Sb-Sc galaxies yields a disk mass that provides $85 \pm 10 \%$ of the total rotation support at $2.2 h_{R}$, where the disk support peaks. Combining this definition of maximal with new observational constraints on Galactic structure parameters leads to the conclusion that it is plausible — and perhaps even likely that the Galactic disk is maximal, in the sense that the dark halo provides negligible rotation support inside two disk scale lengths. In particular, given the new Galactic constraints, the local disk column can be substantially less than the oft-quoted required $\Sigma_{\odot} \approx 100 \mathrm{M}_{\odot} \mathrm{pc}^{-2}$ and still be maximal. The maximal disk models presented here have mass-to-light ratios that lie precisely in the range determined for maximal disks in external Sb-Sc spirals.

On the other hand, if the disk scale length for the Milky Way could be shown definitively to be above $4 \mathrm{kpc}$, most thin maximal disk models would be ruled out. Obtaining tight constraints on the Galactic disk scale length, solar distance, and rotation curve is thus as important as constraining the local surface mass density in assessing the dynamical dominance of the disk in the inner Galaxy.

A Milky Way maximal disk would alter estimates for Galactic dark halo parameters, since the two combined (with the addition of the bar/bulge) give rise to the Galaxy's rotation curve. Any astronomical conclusion, therefore, based on assumed potentials for the disk or dark halo of the Galaxy may need to be revised in light of the possibility that the Galactic disk is maximal. In particular, theoretical estimates for the microlensing optical depth along both the Galactic Center and Magellanic Clouds lines of sight may be in need of revision: a maximal disk would, in general, result in a higher optical depth toward the bulge due to disk lenses, and - since the halo would then be a less dominant component of the inner Galaxy — a lower optical depth along both sight lines due to halo lenses.

It is a pleasure to thank Tjeerd van Albada, Andy Gould, and Konrad Kuijken for useful comments and a critical reading of the manuscript. This work was partially funded by the National Science Foundation (AST 92-15485). 


\section{REFERENCES}

Backer, D.C. 1996, Unsolved Problems of the Milky Way, IAU 169 (Dordrecht: Kluwer) 193

Bahcall, J.N. 1984, ApJ, 276, 169

Bahcall, J.N., Flynn, C. \& Gould, A. 1992, ApJ, 389, 234

Bahcall, J.N. \& Soneira, R. M. 1980, ApJS, 44, 73 (BS)

Beaulieu, J.-P. et al. 1996, A\&Ain press, astro-ph/9612215

Begeman, K., 1987, Ph. D. thesis, University of Groningen, The Netherlands

Binney, J. \& Tremaine, S. 1987, Galactic Dynamics, (Princeton: Princeton Univ. Press)

Broeils, A. 1992, Ph. D. thesis, University of Groningen, The Netherlands

Broeils, A. H. \& Courteau, S. 1997, Dark and Visible Matter in Galaxies and Cosmological Implications, eds. M. Persic \& P. Salluci (San Francisco:ASP), and astro-ph/9610264

Casertano, S. 1983, MNRAS, 203, 735

Casertano, S. \& van Albada, T.S. 1990, Baryonic Dark Matter, eds. D. Lynden-Bell \& G. Gilmore (Dordrecht: Kluwer), 159

Courteau, S. 1996, ApJS, 103, 363.

de Jong, R.S. 1996, A\&A, 313, 45

de Vaucouleurs, G. \& Pence, W.D. 1978, AJ, 83, 1163

Fich, M. \& Tremaine, S. 1991, ARA\&A, 29, 409.

Flynn, C. \& Fuchs, B. 1994, MNRAS, 270, 471

Freeman, K.C. 1993, Physics of Nearby Galaxies: Nature or Nurture? eds. R.X. Thuan, C. Balkowski \& J. Thanh Van, Editions Frontières, 201

Fux, R. \& Martinet, L. 1994, A\&A, 287L, 21

Gould, A., 1990, MNRAS, 244, 25

Gould, A., 1994, ApJ, 426, 542

Gould, A., Bahcall, J.N. \& Flynn, C. 1996, ApJ, 465, 759 
Jacoby, G.H. et al. 1992, PASP, 104, 599

Kent, Dame, T. M. \& Fazio G. 1991, ApJ, 378, 131

Kerr, F.J. \& Lynden-Bell, D. 1986, MNRAS, 221, 1023

Kuijken, K. \& Gilmore, G. 1989a, MNRAS, 239, 571

Kuijken, K. \& Gilmore, G. 1989b, MNRAS, 239, 605

Kuijken, K. \& Gilmore, G. 1991, ApJ, 367, L9 (KG)

Kuijken, K. \& Tremaine, S. 1994, ApJ, 421, 178

Kuijken, K. 1995, Stellar Populations, IAU 164, (Dordrecht: Kluwer), 195

Merrifield, M.R. 1992, AJ, 103, 1552

Merrifield, M.R. 1993, Back to the Galaxy, eds. S.S. Holt \& F. Verter (New York:AIP) 437

Mihalas, D. \& Binney, J. 1981, Galactic Astronomy (San Francisco: Freeman)

Ng, Y.K., Bertelli, G., Bresson, A., Chiosi, C. \& Lub, J. 1995, A\&A, 295, 655

Ojha, D.K., Bienaymé, O., Robin, A.C., Crézé, M. \& Mohan, V. 1996, A\&A, 311, 456

Pierce, M.J. \& Tully, R.B. 1988, ApJ, 330, 579

Pont, F., Mayor, M. \& Burki, G. 1994, A\&A, 285, 415

Pont, F., Queloz, D., Mayor, M. \& Brataschi, P. 1996, Unsolved Problems of the Milky Way, IAU 169 (Dordrecht: Kluwer) 661

Reid, M. 1993, ARA\&A, 31, 345

Reid, N.I. \& Majewski, S.R. 1993, ApJ, 409, 635

Robin, A. C., Crézé, M. \& Mohan, V. 1992, A\&A, 265, 32

Robin, A.C., Haywood, M., Crézé, M., Ojha, D.K., \& Bienaymé, O. 1996, A\&A, 305, 125

Rohlfs, K., Chini, R., Wink, J.E. \& Böhme, R. 1986, A\&A, 158, 181

Sackett, P. D. 1996, Astrophysical Applications of Gravitational Lensing, IAU 173, eds. C.S. Kochanek \& J.N. Hewitt (Dordrecht: Kluwer) 165

Schechter, P. 1993, Back to the Galaxy, eds. S.S. Holt \& F. Verter (New York:AIP) 571 
Sellwood, J.A. \& Sanders, R.H. 1988, MNRAS, 233, 611

Stothers, R.B., 1988, ApJ, 329, 712.

van Albada, T.S. \& Sancisi, R. 1986, Phil. Trans. R. Soc. Lond., A320, 447

van der Kruit, P.C. 1986, A\&A, 157, 230

van der Kruit, P.C. 1989, The Milky Way as a Galaxy, SAAS-FEE 1989, eds. R. Buser \& I. King (Geneva: Geneva Obs.) 185

van der Kruit, P.C. 1995, Stellar Populations, IAU 164, (Dordrecht: Kluwer) 205

Zhao, H.-S., Spergel, D.N. \& Rich, R.M. 1995, ApJ, 440, L13 
Table 1. Recent Determinations of Galactic Scale Parameters with assumed $R_{o}$

\begin{tabular}{lccccc}
\hline \hline \multicolumn{1}{c}{ Reference } & Disk Type & $\begin{array}{c}h_{R} \\
(\mathrm{kpc})\end{array}$ & $\begin{array}{c}h_{z} \\
(\mathrm{kpc})\end{array}$ & $\begin{array}{c}R_{o} \\
(\mathrm{kpc})\end{array}$ & $R_{o} / h_{R}$ \\
\hline Kent, Dame \& Fazio 1991 & 2.4 micron & $3.0 \pm 0.5$ & $0.247^{\mathrm{a}}$ & 8.0 & 2.7 \\
Robin, Crézé, \& Mohan 1992 & thin & $2.5 \pm 0.3$ & constant & 8.5 & 3.4 \\
Fux \& Martinet 1994 & thin & $2.5_{-0.6}^{+0.8}$ & constant $^{\prime}$ & 8.5 & 3.4 \\
Fux \& Martinet 1994 & thin & 3.1 & flaring & 8.5 & 2.7 \\
Ojha et al. 1996 & thin & $2.3 \pm 0.6$ & $0.26 \pm 0.05$ & 8.1 & 3.5 \\
Ojha et al. 1996 & thick & $3 \pm 1$ & $0.76 \pm 0.05$ & 8.1 & 2.7 \\
Robin et al. 1996 & thick & $2.8 \pm 0.8$ & $0.76 \pm 0.05$ & 8.5 & 3.0 \\
Gould, Bahcall \& Flynn 1996 & M-dwarf & $3.0 \pm 0.4$ & $0.45^{\mathrm{c}}$ & 8.0 & 2.7 \\
Reid \& Majewski 1993 & M-dwarf & - & 0.4 & - & - \\
Reid \& Majewski 1993 & thick & - & $1.5 \pm 0.1$ & - & - \\
\hline
\end{tabular}

a at $R_{o}$, and decreasing inwards to $0.165 \mathrm{kpc}$ at the Galactic center

bassumed to rise with $R$ at rate of $30 \mathrm{pc} / \mathrm{kpc}$

'best fit with single component; two components provide better fit 


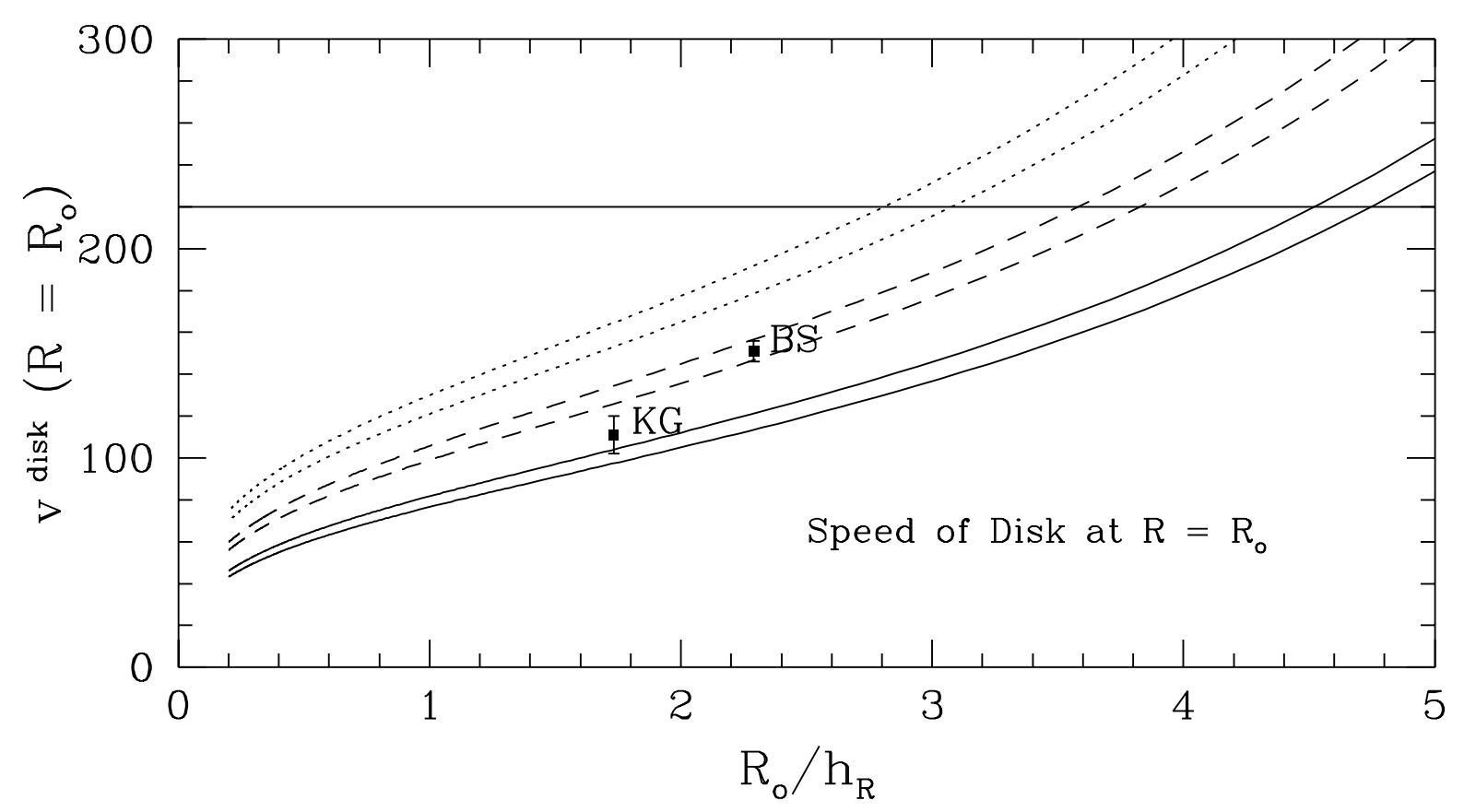

Fig. 1.- The rotation support at the solar position supplied by the disk alone as a function of $R_{o} / h_{R}$ for three disk mass models that are explained more fully in the text: (a) very thin, very light disk (solid lines), (b) moderately thin, heavy disk (dashed lines), and (c) thick, heavy disk (dotted lines). For each mass model, two assumptions for the solar distance are indicated: the lower lines in each pair assume $R_{o}=7.5 \mathrm{kpc}$; the upper lines take $R_{o}=8.5 \mathrm{kpc}$. Any point along these curves represents a particular choice for the disk mass model and the structure constants $R_{o}$ and $h_{R}$. The Galactic disk models of Kuijken \& Gilmore (1991, KG) and Bahcall \& Soneira (1980, BS) are shown. The horizontal line indicates the current IAU standard for the local circular velocity $v_{o}=220 \mathrm{~km} \mathrm{~s}^{-1}$; new measurements indicate that this may be too high. 


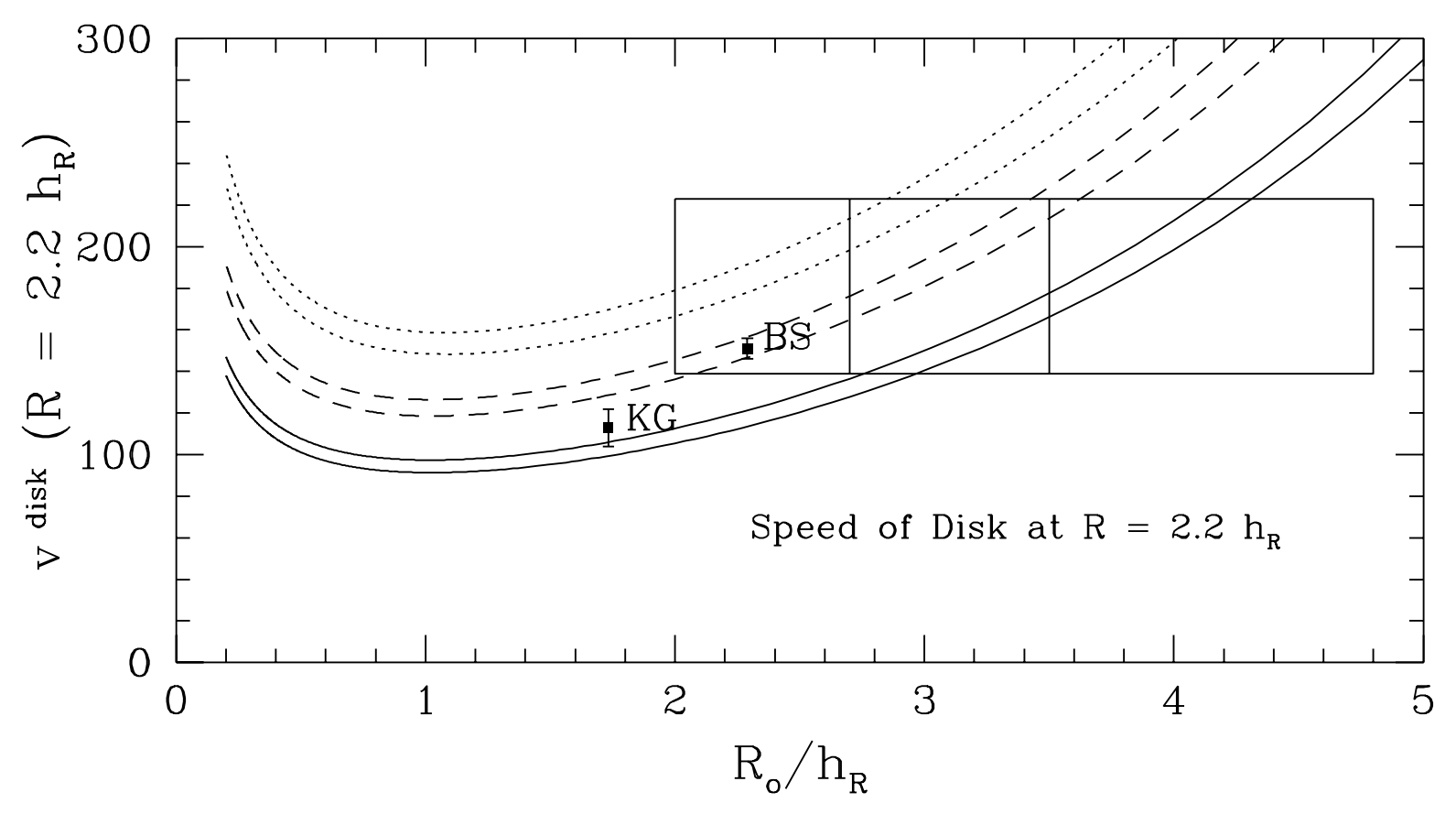

Fig. 2.- The rotation support at $R=2.2 h_{R}$ supplied by the disk alone (at the position of maximum disk support) as a function of $R_{o} / h_{R}$ for the same three models displayed in Fig. 1. The long narrow box encloses the maximum disk constraint for the Galaxy; disk models falling inside the box can be considered to be maximal and satisfying current constraints on $R_{o} / h_{R}$ (see text). The smaller box delineates the range of best fit values for $R_{o} / h_{R}$ from Table 1. 


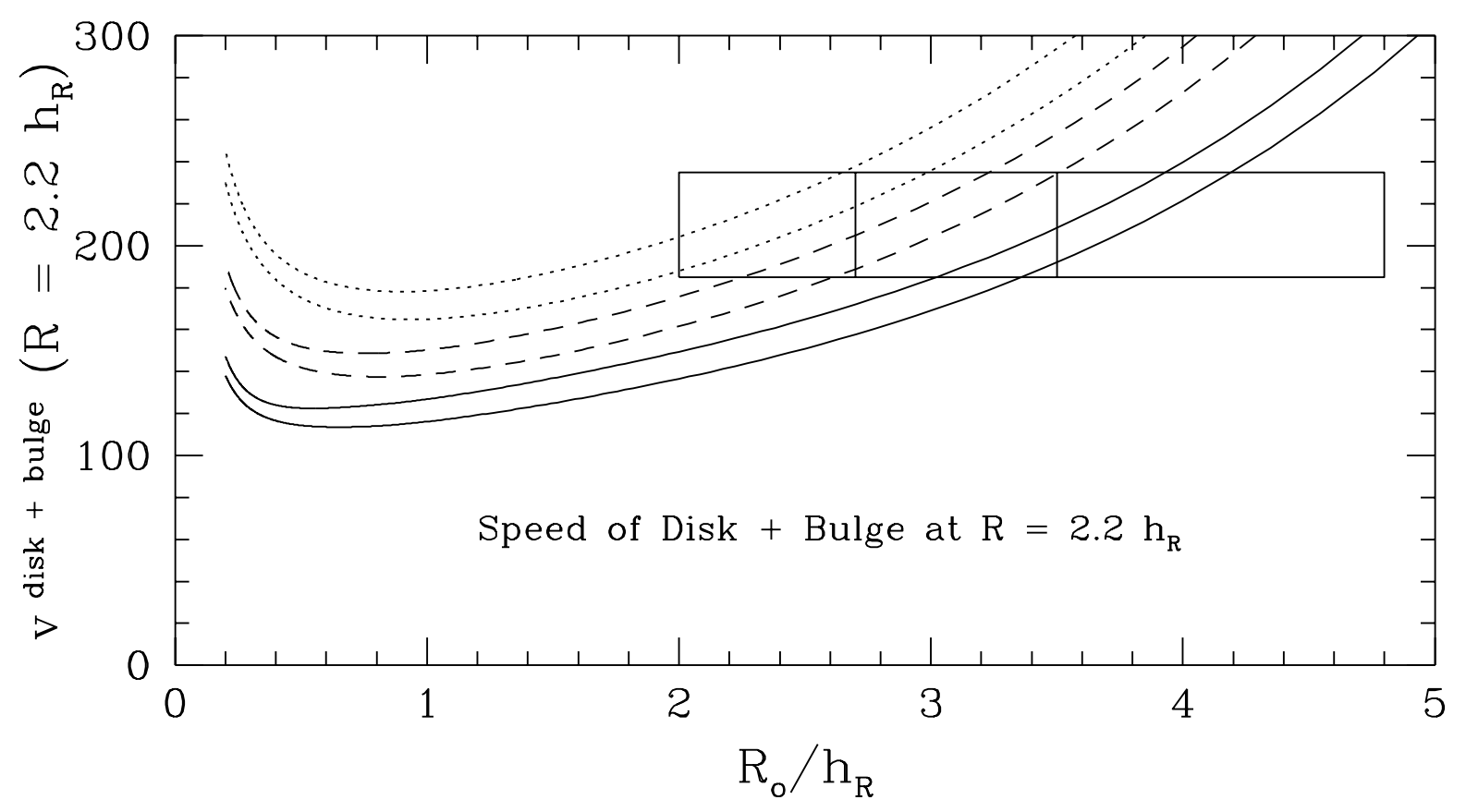

Fig. 3. - The rotation support at $R=2.2 h_{R}$ supplied by the disk and bulge combined as a function of $R_{o} / h_{R}$ for the same three models displayed in Fig. 1. The simple illustrative model for the bulge is explained more fully in text, and is scaled to have the same observed luminosity for all $R_{o}$, giving a range of $1.2 \times 10^{10}<M_{\text {bulge }}<1.8 \times 10^{10} \mathrm{M}_{\odot}$. The long narrow box encloses a strict "maximum luminous mass" constraint for the Galaxy: mass models that fall inside the box satisfy constraints on $R_{o} / h_{R}$, and together the disk and bulge provide all the rotation support at $R=2.2 h_{R}$. The smaller box delineates the range of best fit values for $R_{o} / h_{R}$ from Table 1 . 